\title{
Scalar radiation emitted from a rotating source around a Reissner-Nordström black hole
}

\author{
Luís C. B. Crispind* \\ Faculdade de Física, Universidade Federal do Pará, 66075-110, Belém, PA, Brazil \\ André R. R. da Silvađ and George E. A. Matsa: \\ Instituto de Física Teórica, Universidade Estadual Paulista, \\ Rua Pamplona 145, 01405-900, São Paulo, SP, Brazil
}

(Dated: October 24, 2018)

\begin{abstract}
We investigate the radiation emitted from a scalar source in circular orbit around a ReissnerNordström black hole. Particle and energy emission rates are analytically calculated in the low- and high-frequency regimes and shown to be in full agreement with a numerical calculation. Our investigation is connected with the recent discussion on the validity of the cosmic censorship conjecture in the quantum realm.
\end{abstract}

PACS numbers: 04.62.+v, 04.20.Dw, 41.60.-m

\section{INTRODUCTION}

This is currently under investigation if asymptotically flat spacetimes endowed with some suitable matter content evolved through Einstein equations from some generic initial conditions can give rise to spacetimes with naked singularities (see, e.g., Refs. 1, 2, 3, 4, 5, 6] and references therein). According to the cosmic censorship conjecture (CCC) put forward by Penrose in 1969 such naked singularities should never occur [7]. The validity of the various versions of the CCC is presently source of intense debate (see, e.g., Refs. [8, 9, 10, 11] for comprehensive accounts).

In contrast to naked singularities, the ones present in the interior of black holes are dressed by event horizons. According to the uniqueness theorems, all stationary black hole solutions of the Einstein-Maxwell equations are uniquely determined by the gravitational mass $M$, electric charge $Q$, and angular momentum $J$ satisfying the relationship $M^{2} \geq Q^{2}+(J / M)^{2}$. (We assume natural units $c=G=\hbar=1$ unless stated otherwise.) If we were able to violate the inequality above by overcharging and/or overspinning a black hole, then we would end up with a naked singularity characterized by $Q^{2}+(J / M)^{2}>M^{2}$. Because stationary black holes are stable under linear perturbations [12, 13, 14, 15], they would be a good testing ground for such a search. In 1974, Wald wondered whether or not an extreme black hole $M^{2}=Q^{2}+(J / M)^{2}$ could be overcharged or overspined by the absorption of a classical particle with some proper angular momentum and/or charge [16]. He eventually shows that in order to overcome the gravitational barrier, the classical particle must have just enough energy to compensate any angular momentum and/or

\footnotetext{
*Electronic address: crispino@ufpa.br

${ }^{\dagger}$ Electronic address: dasilva@ift.unesp.br

${ }^{\ddagger}$ Electronic address: matsas@ift.unesp.br
}

charge carried into the hole (see also Ref. [17]). As a result, the black hole constraint $M^{2} \geq Q^{2}+(J / M)^{2}$ would be preserved and the CCC upheld. This illustrates the current belief that classical mechanisms are not expected to undress the singularity hidden in the interior of black holes [18].

On the other hand, it is well known that quantum mechanics can sometimes jeopardize classical assumptions leading to opposite conclusions with respect to the ones obtained in the classical realm. Working in the context of quantum field theory in curved spacetime (QFTCS) Hawking, e.g., was able to show that black holes can evaporate [19] in contrast to the overspread generalrelativistic belief that this would be impossible. It seems natural, then, to inquire as to whether quantum mechanics would have something to add to the CCC issue. An investigation on these lines was recently performed by Ford and Roman [20] who analyzed the possibility of producing a naked singularity by injecting negative energy into an extreme charged black hole. They concluded that the positive energy flux which always follows the negative one would render the CCC true. More recently 21] it was wondered whether or not a scalar wave with small energy but large enough angular momentum could tunnel through the gravitational scattering potential of a nearly extreme macroscopic Reissner-Nordström black hole, $|Q| / M \lesssim 1$, whereby it would acquire enough angular momentum to overspin, $Q^{2}+(J / M)^{2}>M^{2}$, and therefore challenge the CCC. The generalized second law of thermodynamics could still be preserved if the initial entropy of the black hole were carried away by the degrees of freedom of some final debris assuming that naked singularities are unstable. Although a first approximation calculation shows that this is possible, a further analysis performed by Hod 22] suggests that the backreaction on the background spacetime due to the wave angular momentum would preclude it of being absorbed rendering the CCC true again. This is interesting because up to the present knowledge there is no mandatory reason to preclude the formation of naked singularities in the quan- 
tum realm. It is largely believed that quantum gravity should be able to unveil the physical structure of these "entities" making them nonsingular and recovering the spacetime predictability. Indeed, quite recently Richartz and Saa 23] have argued that the quantum overspinning mechanism can be rendered true by replacing the scalar by a fermionic field. This is so because in contrast to the scalar field the fermionic one would not be superradiated away. Probably a final veredictum on the validity of CCC will not be possible before a full quantum formulation of gravity is achieved. In the meanwhile, however, QFTCS can bring us useful insights about this issue.

Here we realize a production mechanism of ingoing and outgoing scalar particles towards and from the ReissnerNordström black hole, respectively, by considering a monopole in circular orbit around the hole coupled to a massless Klein-Gordon field. Refs. [21, 22] assume scalar particles beamed towards the hole but do not mention how they would be produced. Because here we will be mostly interested in waves with small energy and angular momentum in comparison to the hole mass, no spacetime backreaction considerations are in order. This investigation is not only interesting in connection with Refs. 21, 22 but also fulfills a gap in the literature on the so called gravitational synchrotron radiation initiated in the 70's by Misner and collaborators 24] and followed up to these days by a number of authors 25], since scalar radiation emitted from sources around black holes when they are endowed with electric charge has not been considered yet. The usual flat spacetime synchrotron signature can be seen when the monopole moves fast enough. We work in the context of standard QFTCS (see Refs. [26, 27] for comprehensive accounts). Because of the difficulty to express the solution of some differential equations which we deal with in terms of known special functions, our computations are performed (i) numerically but without further approximations and (ii) analytically but restricted to the low- and high-frequency regimes. The paper is organized as follows. In section

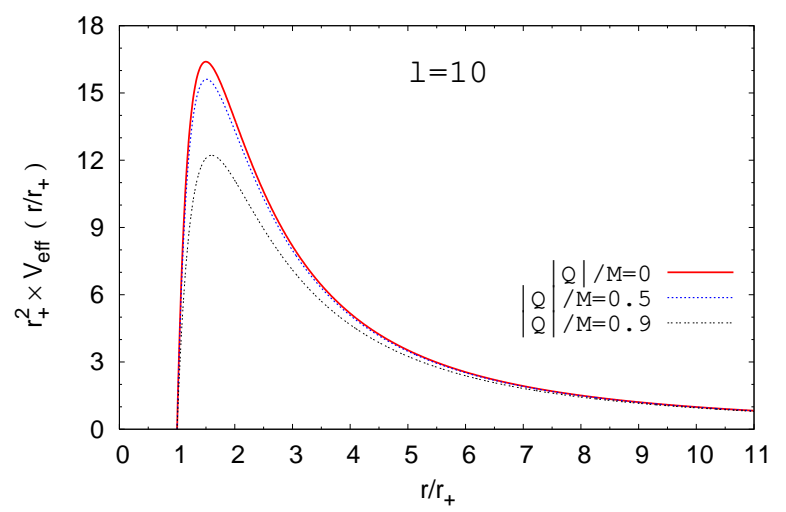

FIG. 1: The effective scattering potential for $|Q| / M=$ $0,0.5$ and 0.9 is plotted for $l=10$ as a function of $r / r_{+}$. The larger the $|Q| / M$ the smaller the $V_{\text {eff }}\left(r / r_{+}\right)$.
II] we present the general formulas for the emission rate and radiated power of scalar particles from the monopole source in circular orbit around the Reissner-Nordström black hole. In section [II] we present analytic results in the low- and high-energy regimes. In section IV the analytic results obtained in the previous section are plotted against a full numerical calculation and shown to agree. We also compute the amount of the emitted radiation which reaches asymptotic observers rather than being absorbed by the hole. Section $\nabla$ is dedicated to our final remarks.

\section{EMISSION RATES AND RADIATED POWERS}

The line element of a Reissner-Nordström black hole with mass $M$ and electric charge $|Q| \leq M$ can be written as [8]

$$
d s^{2}=f(r) d t^{2}-f(r)^{-1} d r^{2}-r^{2}\left(d \theta^{2}+\sin ^{2} \theta d \varphi^{2}\right),
$$

where

$$
f(r) \equiv\left(1-r_{+} / r\right)\left(1-r_{-} / r\right)
$$

and $r_{ \pm} \equiv M \pm \sqrt{M^{2}-Q^{2}}$. Outside the outer event horizon, i.e. for $r>r_{+}$, we have a global timelike isometry generated by the Killing field $\partial_{t}$.

Now we introduce a free massless scalar field $\Phi=$ $\Phi\left(x^{\mu}\right)$ satisfying $\square \Phi=0$. The corresponding field operator can be expanded in terms of creation $a_{\omega l m}^{\alpha \dagger}$ and annihilation $a_{\omega l m}^{\alpha}$ operators as

$$
\hat{\Phi}\left(x^{\mu}\right)=\sum_{\alpha=\leftarrow}^{\rightarrow} \sum_{l=0}^{\infty} \sum_{m=-l}^{l} \int_{0}^{\infty} d \omega\left[u_{\omega l m}^{\alpha}\left(x^{\mu}\right) a_{\omega l m}^{\alpha}+\text { H.c. }\right],
$$

where the normal modes are written as

$$
u_{\omega l m}^{\alpha}=\sqrt{\frac{\omega}{\pi}} \frac{\psi_{\omega l}^{\alpha}(r)}{r} Y_{l m}(\theta, \varphi) e^{-i \omega t}
$$

and are assumed to be orthonormalized according to the Klein-Gordon inner product [26]. Here $\omega \geq 0$ and $l \geq 0$, $m \in[-l, l]$ are frequency and angular momentum quantum numbers, respectively, and $\alpha=\leftarrow(\rightarrow)$ labels ingoing (outgoing) modes. $Y_{l m}(\theta, \varphi)$ are the usual spherical harmonics. $\psi_{\omega l}^{\overleftarrow{\omega l}}(r)$ and $\psi_{\omega l}(r)$ are associated with purely incoming modes from the past null infinity $\mathcal{J}^{-}$and outgoing from the past white-hole horizon $\mathcal{H}^{-}$, respectively. $\psi_{\omega l}^{\alpha}$ satisfies

$$
\left[-f(r) \frac{d}{d r}\left(f(r) \frac{d}{d r}\right)+V_{\mathrm{eff}}(r)\right] \psi_{\omega l}^{\alpha}(r)=\omega^{2} \psi_{\omega l}^{\alpha}(r),
$$

where

$$
V_{\mathrm{eff}}(r)=\left(1-\frac{2 M}{r}+\frac{Q^{2}}{r^{2}}\right)\left(\frac{2 M}{r^{3}}-\frac{2 Q^{2}}{r^{4}}+\frac{l(l+1)}{r^{2}}\right)
$$




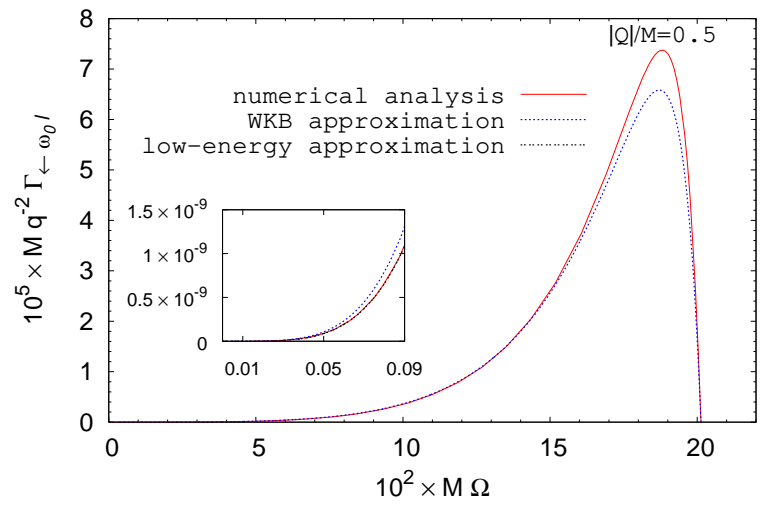

FIG. 2: The numerical result for $\Gamma_{\leftarrow \omega_{0} l}$ with $l=m=5$ is shown assuming a black hole with $|Q| / M=0.5$. The internal box is a zoom of the $\Omega M \ll 1$ region and shows the excellent agreement obtained with our low-energy formulas. The numerical and low-energy results are superimposed and cannot be distinguished with the present resolution. We also plot in this region the result obtained with the WKB method to make it clear that it captures the qualitative behavior in the low-energy region as well. Finally, we emphasize the very nice quantitative approximation provided by the WKB method in the $R_{S} \approx r_{\mathrm{ph}}$ region $(\Omega M \approx 0.2)$.

is the effective scattering potential (see, e.g., Ref. 28] for more detail). A plot of the scattering potential can be found in Fig. 1. The larger the $l$ the larger the $V_{\text {eff }}$ because of the centrifugal barrier. By performing the coordinate transformation

$$
x \equiv y+\frac{\left(y_{+}\right)^{2} \ln \left|y-y_{+}\right|-\left(y_{-}\right)^{2} \ln \left|y-y_{-}\right|}{y_{+}-y_{-}},
$$

where $y \equiv r / 2 M$ and $y_{ \pm} \equiv r_{ \pm} / 2 M$, Eq. (2.5) can be cast in the form

$$
\left(-d^{2} / d x^{2}+4 M^{2} V_{\text {eff }}[r(x)]\right) \psi_{\omega l}^{\alpha}(x)=4 M^{2} \omega^{2} \psi_{\omega l}^{\alpha}(x) .
$$

Accordingly, the creation and annihilation operators satisfy the simple commutation relations

$$
\left[a_{\omega l m}^{\alpha}, a_{\omega^{\prime} l^{\prime} m^{\prime}}^{\alpha^{\prime} \dagger}\right]=\delta_{\alpha \alpha^{\prime}} \delta_{l l^{\prime}} \delta_{m m^{\prime}} \delta\left(\omega-\omega^{\prime}\right)
$$

where the state $|0\rangle$, defined by $a_{\omega l m}^{\alpha}|0\rangle=0$ for every $\alpha, \omega, l$ and $m$, is denominated Boulware vacuum. Close $(x<0,|x| \gg 1)$ to and far away $(x \gg 1)$ from the horizon we have

$$
\psi_{\omega l}^{\overleftarrow{\omega l}}(x) \approx \frac{1}{2 \omega}\left\{\begin{array}{l}
\mathcal{T}_{\omega l}^{\leftarrow} e^{-2 i M \omega x} \quad(x<0,|x| \gg 1) \\
2(-i)^{l+1} M \omega x h_{l}^{(1)}(2 M \omega x)^{*} \\
+2 i^{l+1} \mathcal{R}_{\omega l}^{\leftarrow} M \omega x h_{l}^{(1)}(2 M \omega x) \quad(x \gg 1)
\end{array}\right.
$$

and

$$
\psi_{\omega l}(x) \approx \frac{1}{2 \omega}\left\{\begin{array}{l}
e^{2 i M \omega x}+\mathcal{R}_{\omega l} \overrightarrow{e^{-2 i M \omega x}}(x<0,|x| \gg 1) \\
2 i^{l+1} \mathcal{T}_{\omega l} M \omega x h_{l}^{(1)}(2 M \omega x)(x \gg 1)
\end{array}\right.
$$

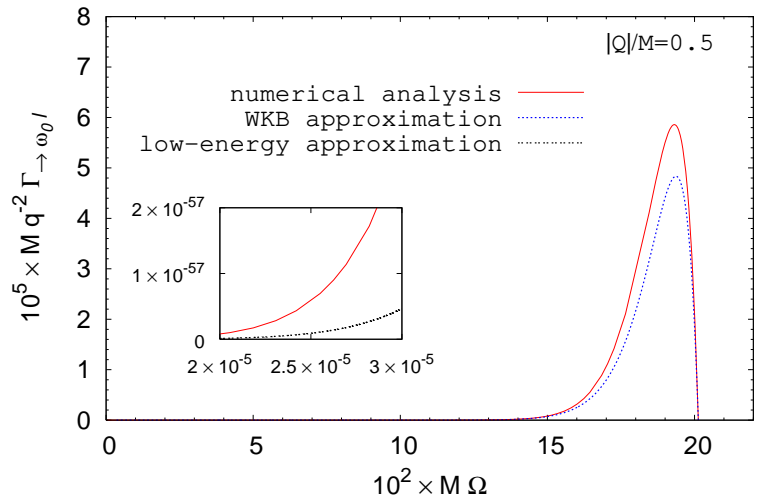

FIG. 3: The numerical result for $\Gamma_{\rightarrow \omega_{0} l}$ with $l=m=5$ and $|Q| / M=0.5$ is plotted. We can see the convergence of the numerical calculation with (i) low-energy analytical results for $\Omega M \ll 1$ and (ii) the WKB method for $R_{S} \approx r_{\mathrm{ph}}(\Omega M \approx 0.2)$.

Here $\left|\mathcal{R}_{\omega l}^{\alpha}\right|^{2}$ and $\left|\mathcal{T}_{\omega l}^{\alpha}\right|^{2}$ are the reflection and transmission coefficients, respectively, satisfying the usual probability conservation equation: $\left|\mathcal{R}_{\omega l}^{\alpha}\right|^{2}+\left|\mathcal{T}_{\omega l}^{\alpha}\right|^{2}=1$ and $h_{l}^{(1)}(2 M \omega x)$ is the spherical Hankel function. Note that $h_{l}^{(1)}(x) \approx(-i)^{l+1} \exp (i x) / x$ for $|x| \gg 1$.

Now let us consider a monopole

$$
j\left(x^{\nu}\right)=\frac{q}{\sqrt{-g} u^{0}} \delta\left(r-R_{S}\right) \delta(\theta-\pi / 2) \delta(\varphi-\Omega t)
$$

describing a scalar source in uniform circular motion at the equatorial plane of the Reissner-Nordström black hole, i.e., $\theta=\pi / 2$, with $r=R_{S}$ and angular velocity $\Omega \equiv d \phi / d t=$ const $>0$ as defined by asymptotic static observers. Here $g \equiv \operatorname{det}\left(g_{\mu \nu}\right)$ and

$$
u^{\mu}\left(\Omega, R_{S}\right)=\left(f\left(R_{S}\right)-R_{S}^{2} \Omega^{2}\right)^{-1 / 2}(1,0,0, \Omega)
$$

is the four-velocity of the source. By assuming that the source is free of interactions other than the gravitational one, we obtain that

$$
\Omega=\sqrt{M / R_{S}^{3}-Q^{2} / R_{S}^{4}},
$$

where

$$
R_{S}>r_{\mathrm{ph}}=\left(3 M+\sqrt{9 M^{2}-8 Q^{2}}\right) / 2 .
$$

Here $r_{\mathrm{ph}}$ is the radius of the null circular geodesic and defines the innermost limit to timelike geodesic circular orbits. We note, moreover, that we have normalized the source $j\left(x^{\mu}\right)$ in Eq. (2.12) by requiring that $\int d \sigma j\left(x^{\mu}\right)=$ $q=$ const, where $d \sigma$ is the proper three-volume element orthogonal to $u^{\mu}$.

Next let us minimally couple the source to the field through the interaction action

$$
\hat{S}_{I}=\int d^{4} x \sqrt{-g} j \hat{\Phi} .
$$


From this we can interpret $q$ in Eq. (2.12) as a coupling constant between source and field. Then the emission amplitude at the tree level of one scalar particle with quantum numbers $(\alpha, \omega, l, m)$ into the Boulware vacuum is given by

$$
\begin{aligned}
\mathcal{A}_{\alpha \omega l m}^{\mathrm{em}} & =\left\langle\alpha \omega l m\left|i \hat{S}_{I}\right| 0\right\rangle \\
& =i \int d^{4} x \sqrt{-g} j\left(x^{\mu}\right) u_{\omega l m}^{\alpha *} .
\end{aligned}
$$

Note that for sources in constant circular motion the amplitude $\mathcal{A}_{\alpha \omega l m}^{\mathrm{em}}$ is proportional to $\delta\left(\omega-\omega_{0}\right)$ where we have defined $\omega_{0} \equiv m \Omega$. Hence the frequency of the emitted particles is constrained by the relation $\omega=\omega_{0}$. In particular, since $\Omega>0$, no waves with $m \leq 0$ are emitted. The emission rate $\Gamma_{\alpha \omega_{0} l}$ and corresponding emitted power $W_{\alpha \omega_{0} l}$ of particles with quantum numbers $\left(\alpha, \omega_{0}, l\right)$ $(l \geq 1)$ are given by

$$
\begin{aligned}
\Gamma_{\alpha \omega_{0} l} & =\int_{0}^{+\infty} d \omega\left|\mathcal{A}_{\alpha \omega l m}^{\mathrm{em}}\right|^{2} / T \\
& =2 q^{2} \omega_{0}\left(f\left(R_{S}\right)-R_{S}^{2} \Omega^{2}\right)\left|\psi_{\omega_{0} l}^{\alpha}\left(R_{S}\right) / R_{S}\right|^{2} \\
& \times\left|Y_{l m}(\pi / 2,0)\right|^{2}
\end{aligned}
$$

and

$$
\begin{aligned}
W_{\alpha \omega_{0} l} & =\int_{0}^{+\infty} d \omega \omega\left|\mathcal{A}_{\alpha \omega l m}^{\mathrm{em}}\right|^{2} / T \\
& =2 q^{2} \omega_{0}^{2}\left(f\left(R_{S}\right)-R_{S}^{2} \Omega^{2}\right)\left|\psi_{\omega_{0} l}^{\alpha}\left(R_{S}\right) / R_{S}\right|^{2} \\
& \times\left|Y_{l m}(\pi / 2,0)\right|^{2},
\end{aligned}
$$

respectively, where $T=2 \pi \delta(0)$ is the total time as measured by asymptotic observers [29]. Note also that $Y_{l m}(\pi / 2,0)=0$ if $l+m$ is odd and

$$
\left|Y_{l m}(\pi / 2,0)\right|^{2}=\frac{2 l+1}{4 \pi} \frac{(l+m-1) ! !(l-m-1) ! !}{(l+m) ! !(l-m) ! !}
$$

if $l+m$ is even 30]. We have defined $n ! ! \equiv n(n-2) \cdots 1$ if $n$ is odd, $n ! ! \equiv n(n-2) \cdots 2$ if $n$ is even and $(-1) ! ! \equiv 1$. Moreover, note that if we had chosen the Unruh or Hartle-Hawking vacua rather than the Boulware one then Eqs. (2.18)-(2.19) would be associated with the net emitted radiation since the absorption and stimulated emission rates (which are induced by the presence of thermal fluxes) are the same.

The total emission rate $\Gamma^{\text {total }}$ and radiated power $W^{\text {total }}$ are obtained by summing on the quantum numbers $\alpha, l, m$ in Eqs. (2.18) and (2.19), accordingly. The total particle and energy rates which escape to infinity are

$$
\Gamma^{\mathrm{obs}}=\sum_{l=1}^{\infty} \sum_{m=1}^{l}\left(\left|\mathcal{T}_{\omega_{0} l}^{\rightarrow}\right|^{2} \Gamma_{\rightarrow \omega_{0} l}+\left|\mathcal{R}_{\omega_{0} l}^{\leftarrow}\right|^{2} \Gamma_{\leftarrow \omega_{0} l}\right)
$$

and

$$
W^{\mathrm{obs}}=\sum_{l=1}^{\infty} \sum_{m=1}^{l}\left(\left|\mathcal{T}_{\omega_{0} l}\right|^{2} W_{\rightarrow \omega_{0} l}+\left|\mathcal{R}_{\omega_{0} l}^{\leftarrow}\right|^{2} W_{\leftarrow \omega_{0} l}\right),
$$
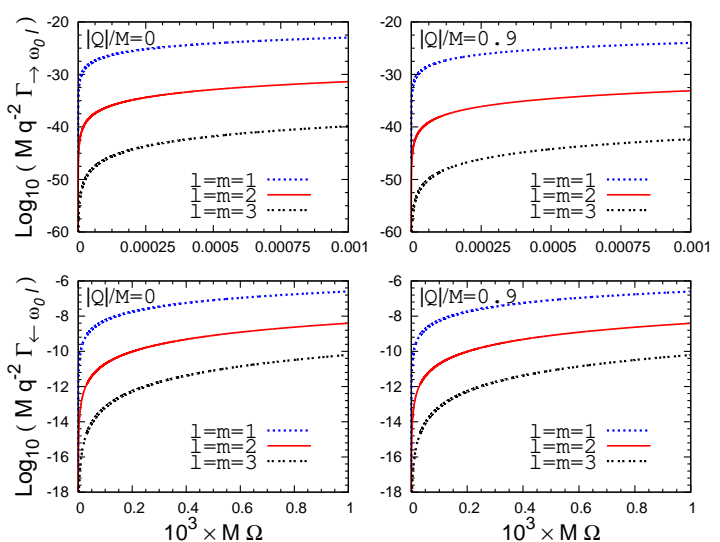

FIG. 4: The particle emission rates $\Gamma_{\alpha \omega_{0} l}(\alpha=\leftarrow, \rightarrow)$ are plotted in the low-frequency regime as a function of the source angular velocity $\Omega$ for different values of $l(m=l) . \Gamma_{\leftarrow \omega_{0} l}$ is seen to be larger than $\Gamma \rightarrow \omega_{0} l$. The larger the $l$ and $|Q| / M$ the smaller the $\Gamma_{\alpha \omega_{0} l}$.

respectively. Here we note that $\mathcal{T}_{\omega_{0} l}^{\leftarrow}=\mathcal{T}_{\omega_{0} l}$. This guaranties that $\left|\mathcal{R}_{\omega_{0} l}^{\leftarrow}\right|=\mid \mathcal{R}_{\omega_{0} l} l$. Note, however, that $\mathcal{R}_{\omega_{0} l}^{\leftarrow}$ and $\mathcal{R}_{\omega_{0} l}$ will in general differ by a phase (in contrast to $\mathcal{T}_{\omega_{0} l}^{\leftarrow}$ and $\left.\underset{\mathcal{T}_{\omega_{0} l}}{\longrightarrow}\right)$.

\section{LOW- AND HIGH-ENERGY SOLUTIONS}

Now, in order to calculate the physical observables given by Eqs. (2.18)-(2.19) and (2.21)-(2.22) we must work out the functions $\psi_{\omega l}^{\alpha}(r)$. We exhibit approximate low- and high-frequency solutions which are going to be
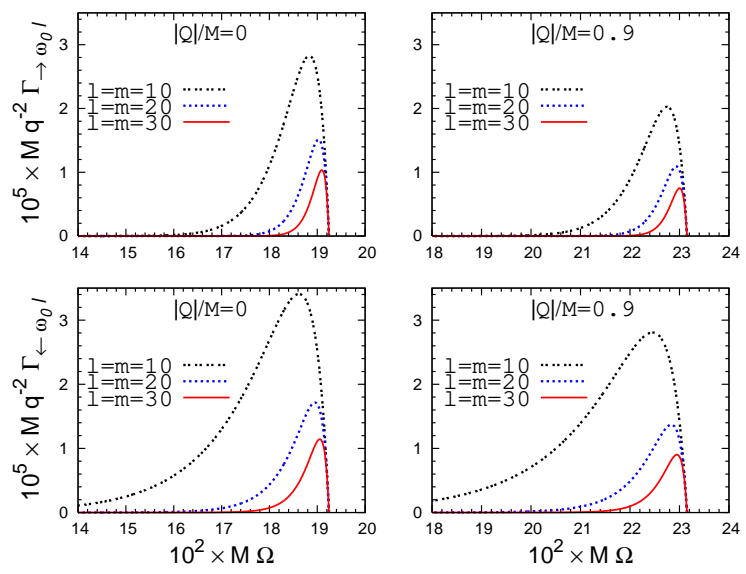

FIG. 5: The particle emission rates $\Gamma_{\alpha \omega_{0} l}$ are plotted in the high-frequency regime for different values of $l(m=l)$. The qualitative behavior of $\Gamma_{\alpha \omega_{0} l}$ are much like the ones observed in the low-frequency regime with one exception, namely, $\Gamma_{\rightarrow \omega_{0} l}$ becomes larger than $\Gamma_{\leftarrow \omega_{0} l}$ for $R_{S} \approx r_{\mathrm{ph}}$. The existence of charge in the black hole makes $\Gamma_{\alpha \omega_{0} l}$ to decrease. The figures are plotted up to the last timelike geodesic circular orbit. 
used in the next section in conjunction with a full numerical calculation designed to cover the whole frequency range.

\section{A. Low-energy solutions}

The low-frequency solution for $\psi_{\omega l}^{\alpha}(r)$ has been already worked out in Ref. 28] and can be cast (up to an arbitrary phase) in the form

$$
\psi_{\omega l}(r)=\frac{-4 i M y_{+} y Q_{l}[z(y)]}{y_{+}-y_{-}}
$$

and

$$
\psi_{\omega l}^{\overleftarrow{\omega}}(r)=\frac{2^{2 l+1}(-i)^{l+1}(l !)^{3} M^{l+1}\left(y_{+}-y_{-}\right)^{l} \omega^{l} y P_{l}[z(y)]}{(2 l+1) !(2 l) !}
$$

where

$$
z(y) \equiv \frac{2 y-1}{y_{+}-y_{-}}
$$

One can also obtain

$$
\mathcal{T}_{\omega l}=\frac{2^{2 l+2}(-i)^{l+1} y_{+}\left(y_{+}-y_{-}\right)^{l}(l !)^{3}(M \omega)^{l+1}}{(2 l+1) !(2 l) !}
$$

in the low-frequency regime. We recall that $\mathcal{T}_{\omega l}^{\overleftarrow{ }}=\mathcal{T}_{\omega l}$. Eq. (3.4) was used in Ref. 21] to calculate the probability $\left|\mathcal{T}_{\omega l}\right|^{2}$ of a wave to tunnel into the black hole assuming a fixed Reissner-Nordström effective scattering potential. The larger the black hole mass and charge in comparison with the wave energy and angular momentum the better the static potential approximation. Here we consider large enough black holes in order to neglect spacetime backreaction effects.

\section{B. High-energy solutions}

A good approximation of $\psi_{\omega l}^{\alpha}(r)$ for high energies can be obtained by using the WKB approximation (see, e.g., Ref. [31]). To do so, it is worth noting that Eq. (2.8) resembles the one-dimensional Schrödinger equation. By considering the effective energy $\omega^{2}$ lower than the peak of $V_{\text {eff }}(x)$, one has two distinct situations. The first one is characterized by $V_{\text {eff }}(x)<\omega^{2}$ which is valid in the intervals $\left(-\infty, x_{-}\right)$and $\left(x_{+},+\infty\right)$, where $x_{ \pm}$ $\left(x_{-}<x_{+}\right)$stands for the classical turning points which satisfy $V_{\text {eff }}\left(x_{ \pm}\right)=\omega^{2}$. Then, in the region where $k_{\omega l}^{-1} d\left(\ln k_{\omega l}\right) / d x \ll 1$ is satisfied, we write down

$$
\psi_{\omega l}^{\overleftarrow{\omega l}}(x) \approx \frac{A_{\omega}^{\leftarrow}}{\sqrt{k_{\omega l}}}\left\{\begin{array}{l}
\mathcal{T}_{\omega l}^{\leftarrow} e^{-i\left(\sigma_{\omega l}-\pi / 4\right)}(x<0,|x| \gg 1) \\
e^{-i\left(\rho_{\omega l}+\pi / 4\right)} \\
+\mathcal{R}_{\omega l}^{\leftarrow} e^{i\left(\rho_{\omega l}+\pi / 4\right)}(x \gg 1)
\end{array}\right.
$$

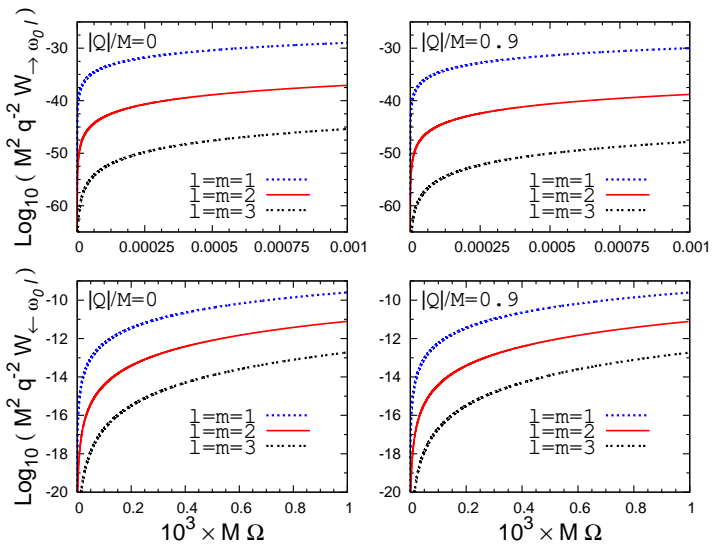

FIG. 6: The radiated powers $W_{\alpha \omega_{0} l}(\alpha=\leftarrow, \rightarrow)$ are plotted in the low-frequency regime as a function of the source angular velocity $\Omega$ for different values of $l(m=l)$. The qualitative behavior of $W_{\alpha \omega_{0} l}$ and $\Gamma_{\alpha \omega_{0} l}$ are similar to each other in the low-frequency regime in contrast to what we see in the highfrequency one.

and

$$
\psi_{\omega l}(x) \approx \frac{A_{\omega}}{\sqrt{k_{\omega l}}}\left\{\begin{array}{l}
\mathcal{R}_{\omega l} e^{-i\left(\sigma_{\omega l}-\pi / 4\right)} \\
+e^{i\left(\sigma_{\omega l}-\pi / 4\right)}(x<0,|x| \gg 1) \\
\mathcal{T}_{\omega l} e^{i\left(\rho_{\omega l}+\pi / 4\right)}(x \gg 1)
\end{array}\right.
$$

where we have defined

$$
\sigma_{\omega l}(x) \equiv \int_{x_{-}}^{x} k_{\omega l}\left(x^{\prime}\right) d x^{\prime}
$$

and

$$
\rho_{\omega l}(x) \equiv \int_{x_{+}}^{x} k_{\omega l}\left(x^{\prime}\right) d x^{\prime}
$$
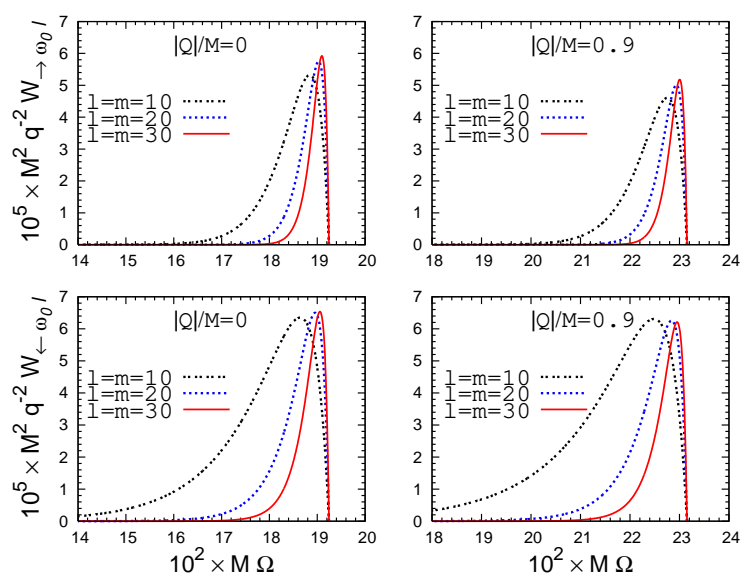

FIG. 7: The radiated powers $W_{\alpha \omega_{0} l}(\alpha=\leftarrow, \rightarrow)$ are plotted in the high-frequency regime. Note that depending on the value of $\Omega$ different $l$ values contribute the most. 
with $k_{\omega l}(x) \equiv 2 M \sqrt{\omega^{2}-V_{\text {eff }}(x)}$. Here the normalization constants $A_{\omega}^{\alpha}$ are determined by an asymptotic fitting between Eqs. (2.10)-(2.11) and Eqs. (3.5)-(3.6), respectively. As a result, we obtain $\left|A_{\omega}^{\leftarrow}\right|=\left|A_{\omega}\right|=\sqrt{M / 2 \omega}$.

Now, we must analyze the case $V_{\text {eff }}(x)>\omega^{2}$, which occurs in the interval $\left(x_{-}, x_{+}\right)$. In this region, $\psi_{\omega l}^{\alpha}(r)$ can be cast in the form

$$
\psi_{\omega l}^{\overleftarrow{\omega l}}(x) \approx-i \frac{A_{\omega}^{\leftarrow}}{\sqrt{\kappa_{\omega l}}} e^{-\xi_{\omega l}}
$$

and

$$
\psi_{\omega l}(x) \approx-i \frac{A_{\omega}}{\sqrt{\kappa_{\omega l}}} e^{\left(\Theta_{\omega l}+\xi_{\omega l}\right)}
$$

assuming $\kappa_{\omega l}^{-1} d\left(\ln \kappa_{\omega l}\right) / d x \ll 1$, where we have defined

$$
\xi_{\omega l}(x) \equiv \int_{x}^{x_{+}} \kappa_{\omega l}\left(x^{\prime}\right) d x^{\prime}
$$

and

$$
\Theta_{\omega l} \equiv-\int_{x_{-}}^{x_{+}} \kappa_{\omega l}(x) d x
$$

with $\kappa_{\omega l}(x) \equiv 2 M \sqrt{V_{\text {eff }}(x)-\omega^{2}}$. The quantity $\Theta_{\omega l}$ is the well-known barrier factor and is associated with the transmission coefficient by

$$
\left|\mathcal{T}_{\omega l}^{\alpha}\right|^{2} \approx e^{2 \Theta_{\omega l}} .
$$

As a consequence, $\left|\mathcal{R}_{\omega l}^{\alpha}\right|^{2} \approx 1-e^{2 \Theta_{\omega l}}$.

\section{Numerical calculation}

Now, in order to plot particle and energy emission rates in the whole frequency range, a numerical calculation procedure is in order. Briefly speaking the numerical method consists of solving Eq. (2.8) for the leftand right-moving radial functions $\psi_{\omega_{0} l}^{\overleftarrow{ }}(r)$ and $\psi_{\omega_{0} l}(r)$ with asymptotic boundary conditions compatible with Eqs. (2.10) and (2.11), respectively. We address to Ref. 32] for more detail.

\section{RESULTS}

In Figs. 2 and 3 we show the particle emission rates for $l=m=5$, and $\alpha=\leftarrow$ and $\rightarrow$, respectively. We also display a zoom for $M \Omega \ll 1$. We can see the good approximation provided by our low-energy formulas, which are applicable when the source is in circular orbits far away from the horizon. In the same token, the results obtained using the WKB approximation reproduce very well the curves for $R_{S} \approx r_{\mathrm{ph}}$, i.e., when the source is close to the innermost timelike geodesic circular orbit. This is the region where most emitted particles are high-energy

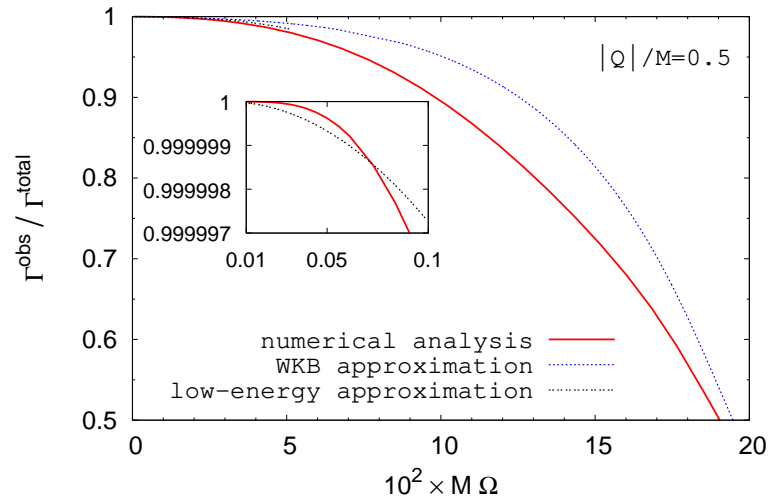

FIG. 8: We plot the rate of particles which reach asymptotic observers as a function of $\Omega$ for $|Q| / M=0.5$. The sum in Eq. (2.21) is taken up to $l=5$. It is seen that the WKB and the low-energy approximation give good results in the proper regions, as expected. It is interesting to note that for $R_{S} \approx r_{\mathrm{ph}}(\Omega M \approx 0.2)$ about half of the emitted particles are lost inside the hole.

ones. This is also convenient to notice that the WKB approximation reproduces most qualitative aspects of the exact numerical calculation. The WKB approximation is specially good for large angular momentum quantum numbers $l$, as expected. This is very handy when dealing with large $l$ solutions since the WKB method requires comparatively modest computational resources in contrast to the full numerical procedure. In Figs. 4 and 5 we analyze in more detail the low- and high-energy particle emission regions by using the proper formulas, namely, Eq. (2.18) with Eqs. (3.1)-(3.2) and Eqs. (3.7)-(3.8), respectively. We note that $\Gamma_{\leftarrow \omega_{0} l}$ is typically larger than $\Gamma_{\rightarrow \omega_{0} l}$ except for $R_{S} \approx r_{\mathrm{ph}}$ and that the larger the $l$ the smaller the $\Gamma_{\alpha \omega_{0} l}$. (For a fixed $l$ the larger the $m$ the larger the contribution provided that $l+m$ is even.) Moreover the presence of charge in the black hole tends to damp $\Gamma_{\alpha \omega_{0} l}$. In Figs. [6 and 7 we analyze in more detail the radiated power in the regions where the source is far

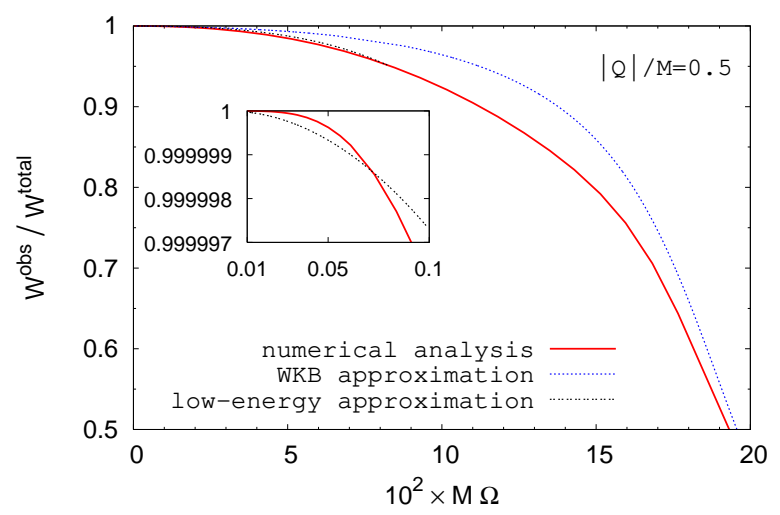

FIG. 9: The power observed at infinity is plotted as a function of $\Omega$ for $|Q| / M=0.5$. 
away from the horizon and close to the innermost timelike geodesic orbit by using Eq. (2.19) with Eqs. (3.1)- (3.2) and Eqs. (3.7)-(3.8), respectively. Far away from the hole the leading contribution to the power comes from the mode with $l=m=1$, while for $R_{S} \approx r_{\text {ph }}$ this will depend on the source angular velocity $\Omega$. In Figs. 8 and 9 we plot the particle emission rate and corresponding power which reach asymptotic observers for $|Q| / M=0.5$, respectively. It is worth noting that for $R_{S} \approx r_{\mathrm{ph}}$ about half of the emitted particles are absorbed by the hole. The WKB and the low-energy approximations are in nice agreement with the numerical results.

\section{FINAL REMARKS}

We have considered the scalar radiation emitted from a monopole in circular geodesic orbits around a charged black hole. Emission rates and radiated powers were calculated using exact numerical and approximate analytical calculations, and shown to be in excellent agreement with each other in the proper regions. The net radiation which reaches asymptotic observers was also investi- gated and shown to decrease up to $50 \%$ when the source is close to the innermost geodesic circular orbit. Because we have only assumed here particles with small angular momentum and energy in comparison to the black hole mass and charge, no significant backreaction effects are expected and, thus, the background spacetime was regarded as fixed. The possibility of challenging the CCC by the tunneling of particles with high enough angular momenta through the black hole scattering potential has been recently discussed [21, 22, 23]. Here we have offered a production mechanism for these particles. Fermionic ones considered in Ref. [23] can be produced by a similar mechanism.

\section{Acknowledgments}

L. C. and G. M. are grateful to Conselho Nacional de Desenvolvimento Científico e Tecnológico ( $\mathrm{CNPq}$ ) for partial financial support. A. S. and G. M. would like also to acknowledge partial and full financial support from Fundação de Amparo à Pesquisa do Estado de São Paulo (FAPESP), respectively.
[1] D. G. Boulware, Phys. Rev. D 8, 2363 (1973).

[2] D. M. Eardley and L. Smarr, Phys. Rev. D 19, 2239 (1979).

[3] C. Christodolou, Comm. Math. Phys. 93, 171 (1984).

[4] P. S. Joshi and I. H. Dwivedi, Phys. Rev. D 47, 5357 (1993).

[5] M. W. Choptuik, Phys. Rev. Lett. 70, 9 (1993).

[6] T. Hertog, G. T. Horowitz and K. Maeda, Phys. Rev. Lett. 92, 131101 (2004).

[7] R. Penrose, Riv. Nuovo Cimento 1, 252 (1969).

[8] R. M. Wald, General Relativity (University of Chicago Press, Chicago, 1984).

[9] C. J. S. Clarke, Class. Quantum Grav. 11, 1375 (1994).

[10] R. M. Wald, arXiv:gr-qc/9710068.

[11] R. Penrose, J. Astrophys. Astr. 20, 233 (1999).

[12] C. V. Vishveshwara, Phys. Rev. D 1, 2870 (1970).

[13] R. Price, Phys. Rev. D 5, 2419 (1972); R. Price, Phys. Rev. D 5, 2439 (1972).

[14] B. S. Kay and R. M. Wald, Class. Quantum Grav. 4, 893 (1987).

[15] B. F. Whiting, J. Math. Phys. 30, 1301 (1989).

[16] R. M. Wald, Ann. Phys. (N.Y.) 82, 548 (1974).

[17] T. Needham, Phys. Rev. D 22, 791 (1980).

[18] S. W. Hawking and G. F. R. Ellis, The Large Structure Scale of Space-Time (Cambridge University Press, Cambridge, England, 1973).

[19] S. W. Hawking, Nature (London) 248, 30 (1974).

[20] L. H. Ford and T. A. Roman, Phys. Rev. D 41, 3662 (1990); L. H. Ford and T. A. Roman, Phys. Rev. D 46, 1328 (1992).

[21] G. E. A. Matsas and A. R. R. da Silva, Phys. Rev. Lett. 99, 181301 (2007).
[22] S. Hod, Phys. Rev. Lett. 100, 121101 (2008).

[23] M. Richartz and A. Saa, arXiv:gr-qc/0804.3921.

[24] C. W. Misner, R. A. Breuer, D. R. Brill, P. L. Chrzanowski, H. G. Hughes III and C. M. Pereira, Phys. Rev. Lett. 28, 998 (1972). R. A. Breuer, P. L. Chrzanowski, H. G. Hughes III and C. W. Misner, Phys. Rev. D 8, 4309 (1973). R. A. Breuer, Gravitational Perturbation Theory and Synchrotron Radiation - Lecture Notes in Physics (Springer-Verlag, Heidelberg, 1975).

[25] E. Poisson, Phys. Rev. D 52, 5719 (1995). L. M. Burko, Phys. Rev. Lett. 84, 4529 (2000). V. Cardoso and J. P. S. Lemos Phys. Rev. D 65, 104033 (2002). J. Castiñeiras, L. C. B. Crispino, R. Murta and G. E. A. Matsas, Phys. Rev. D 71, 104013 (2005).

[26] N. D. Birrel and P. C. W. Davies, Quantum Fields in Curved Space (Cambridge University Press, Cambridge, 1982).

[27] S. A. Fulling, Aspects of Quantum Field Theory in Curved Space-Time (Cambridge University Press, Cambridge, 1989).

[28] J. Castiñeiras and G. E. A. Matsas, Phys. Rev. D 62, 064001 (2000).

[29] C. Itzykson and J.-B. Zuber, Quantum Field Theory (McGraw-Hill, New York, 1980).

[30] I. S. Gradshteyn and I. M. Ryzhik, Tables of Integrals, Series, and Products (Academic Press, New York, 1980).

[31] E. Merzbacher, Quantum Mechanics (John Wiley, New York, 1998).

[32] L. C. B. Crispino, A. Higuchi and G. E. A. Matsas, Class. Quantum Grav. 17, 19 (2000). 